\title{
p 块中不可约模表示的个数
}

$G$ 是有限群, $F$ 是 $G$ 的特征为 $p$ 的分裂域。 $F G$ 是群代数，我们简记为 $R=F G . J(R)$ 是 $R$ 的根. 对 $R$ 的任一子代数 $W$,令 $\tilde{W}=(W+J(R)) / J(R)$, 它 是 $\tilde{R}=R / J(R)$ 的子代数. 特别地当 $w$ 是理想时, $\tilde{W}$ 是 $\tilde{R}$ 的单理想的直和,这时 $\tilde{W}$ 看成 $R$ 模是不可约 $R$ 模的直和(带有血数).

对任一 $F$ 和代数 $A$, 令 $S_{A}=\{a b-b a \mid a, b \in$ A\}, $T_{A}=\{X \in A \mid x)^{m} \in S_{A}, m$ 是某正整数 $\}$.

引玨 设 $A$ 是 $R-F G$ 的任一理想, 则

$$
J(A)=J(R) \cap A
$$

且 $T_{A}=T_{R} \cap A$.

由此引理我们可得到 Brauer 的一个定理(參见 B. Huppert and N. Blackbusn, Finite Groups II, Springer-Verlag, 1982, 第七章定理 3.8) 的深化.

令 $x_{1}=1, x_{2}, \cdots, x_{2}$ 是 $G$ 的全部元素, 而 $C_{1}$, $\cdots, c_{\mathrm{A}}$ 是 $G$ 的全部 $p^{\prime}$ 类. 令

$C^{*}=\left\{x \in G \mid x^{* m} \in C_{j}, m\right.$ 为某非负整数 $\}, j=$ $1, \cdots, k$. 对 $R=F G$ 的一个子代数 $A$, 令 $y_{1}, \cdots, y_{s}$ 是 $A$ 的基. 设

$$
y_{i}=\sum_{i=1}^{b} a_{i}, x_{j}(i=1, \cdots, s),
$$

\section{$n$ 元 全差置 换 的 数目}

，元整换

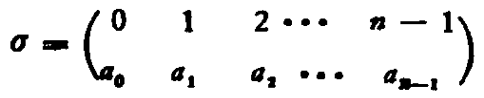

称之为全差置换,如果当 $i \neq j$ 时,有

$$
a-a_{i} \neq j-a_{1}(\bmod n) \text {. }
$$

容易证明, $n$ 元全差置换存在的充分必要条件 是 $n$ 为奇数.

C. Tompkins 在 “Machine atzacks on problems whose variables are permutations"一文中给出了 $n \leqslant$ 11 的全差置换的数目.

本文研究了 $n$ 元全差置换组成的集合上的变换 群,运用群作用在集合上的思想, 并且采用 DFS 算
令

$$
b_{i j}=\sum_{\substack{1<i<i \\ x_{i} \in C_{i}^{*}}} a_{i \ell},
$$

$i=1,2, \cdots, s ; j=1, \cdots, k$. 作矩阵

$$
M(A)=\left(b_{i j}\right)_{s \times k} .
$$

定理 设 $A$ 是 $R=F G$ 的理想, $F$ 是 $G$ 的特 征为 $p$ 的分裂域。 $y_{1}, \cdots, y_{s}, b_{i}, i=1, \cdots, s$; $i=1, \cdots, k$ 及 $M(A)$ 如上. 则由 $\tilde{A}$ (作为 $F G$ 模) 提供的不等价不可约表示的个数等于 $M(A)$ 的 $F$ 秩.

令 $D$ 是 $G$ 的 $p$ 子群. $V_{D}\left(V_{D}^{0}\right)$ 是 $Z(F G)$ 中由有 亏数群 $\subseteq D(\subsetneq D)$ 的共轭类和生成的理想.

推论屈于有亏数群 $\subseteq D$ 及 $\subsetneq D$ 的块的不 可约模表示的个数分别是 $F$ 秩 $M\left(V_{D} R\right)$ 和 $F$ 秩 $M$ $\left(V_{D}^{0} R\right)$.

推论 设 $e$ 是 $R$ 的一个块赛等元，则属于块 $e R$ 的不可约模表示的个数为 $F$ 秩 $M(e R)$.

石生明

（北京大学数学系） 
对于 $n=17 、 19 、 21 、 23$, 运用公式(1)求出的 $\omega_{n}$ 的估值依次是:

$$
\begin{aligned}
& 2.015443 \times 10^{9}, \\
& 1.275789 \times 10^{11}, \\
& 9.9176865 \times 10^{12}, \\
& 9.2884469 \times 10^{14},
\end{aligned}
$$

$\omega_{n}$ 的疍信概率为 $90 \%$ 的置信区间依次是: $\left[1.463591 \times 10^{\circ}, 2.775390 \times 10^{\circ}\right]$,

$\left[8.927731 \times 10^{10}, 1.823124 \times 10^{18}\right]$, $\left[6.658207 \times 10^{12}, 1.477281 \times 10^{13}\right]$, $\left[5.963276 \times 10^{14}, 1.446775 \times 10^{25}\right]$.

兵维声 （北京大学数学系）

\section{蔡查罗奇异积分渐近公式的推广}

\section{我们约定记号}

$$
\begin{aligned}
& S_{*}(f, x)=\frac{1}{\pi} \int_{-*}^{*} f(x+t) D_{\nu}(t) d t, \\
& \tilde{S}_{*}(f, x)=\frac{1}{\pi} \int_{-*}^{*} f(x+t) \tilde{D}_{\nu}(t) d t,
\end{aligned}
$$

其中

$$
\begin{aligned}
& D_{\nu}(t)=\frac{1}{2}+\sum_{\mu=1}^{\nu} \cos \mu t, \\
& \tilde{D}_{\nu}(t)=\sum_{\mu=1}^{\nu} \sin \mu t,
\end{aligned}
$$

井令

$$
A_{n}=\frac{(r+1) \cdots(r+n)}{n !}, A_{n}^{0}=1 .
$$

如果盟

$$
\begin{aligned}
& K_{n}^{*}(t)=\frac{1}{A_{n}^{*}} \sum_{\nu=0}^{n} A_{n=v}^{n}=D_{\nu}(t) ; \\
& \tilde{K}_{n}^{\prime}(t)=\frac{1}{A_{n}^{2}} \sum_{\nu=0}^{n} A_{n=\nu}^{n} \tilde{D}_{\nu}(t), \\
& \sigma_{n}^{*}(x)=\frac{1}{\pi} \int_{-\pi}^{*} f(x+t) K_{n}^{\prime}(t) d t ; \\
& \tilde{\sigma}_{n}^{\prime}(x)=\frac{1}{\pi} \int_{-\pi}^{*} f(x+t) \tilde{K}_{n}^{\prime}(t) d t,
\end{aligned}
$$

这里 $f(x) \in C_{2 x}$. 那么称算子 (2) 为蔡查罗奇异积 分.

设 $f(x) \in \operatorname{Lip}_{1} \alpha$, 也即 $\left|f\left(t_{1}\right)-f\left(t_{2}\right)\right| \leqslant$ $\left|t_{1}-t_{2}\right|^{\alpha}, 0<\alpha \leqslant 1$, 对所有 $t_{1}, t_{2} \in[-\pi, \pi]$.

R. Taberski 在 1962 年给出量

$$
\left.\Delta_{i}^{\prime}(\alpha)=\sup _{\mid \in L p_{1} \alpha} \max _{-\pi<x<\pi}\left|\sigma_{*}^{r}(x)-f(x)\right|\right\}
$$

和

$$
\tilde{\Delta}_{n}^{\prime}(\alpha)=\sup _{1 \in L p_{1}=}\left\{\max _{x<x<\pi}\left|\tilde{\sigma}_{n}^{r}(x)-\tilde{f}(x)\right|\right\}
$$

的渐近表达式,即其中

$$
f(x)=-\frac{1}{2 \pi} \int_{0}^{x}\{f(x+t)-f(x-t)\} \operatorname{ctg} \frac{1}{2} d t
$$

时得到

$$
\begin{aligned}
& \Delta_{:}(\alpha)=\left\{\begin{aligned}
\frac{2}{\pi n^{\alpha}} & c,(\alpha) \Gamma(\alpha) \sin \frac{\alpha \pi}{2} \\
& +o\left(\frac{1}{n}\right), \quad \text { 当 } 0<\alpha<1,
\end{aligned}\right. \\
& \frac{2 r}{n \pi} \ln n+o\left(\frac{1}{n}\right) \text {, 当 } \alpha=1 \text {, }
\end{aligned}
$$

对于 $r=1,2, \cdots$ 成立, 和

$$
\tilde{\Delta}_{i}^{\prime}(\alpha)=\left\{\begin{array}{c}
\frac{1}{\pi}\left(\frac{2}{n}\right)^{\alpha} c_{r}(\alpha) \Gamma(\alpha) \cos \frac{\alpha \pi}{2} \\
+o\left(\frac{\ln n}{n}\right), \quad \text { 当 } 0<\alpha<1, \text { (1) } \\
\frac{r}{n}+o\left(\frac{1}{n^{2}}\right), \quad \text { 当 } \alpha=1,
\end{array}\right.
$$

对于 $r=3,4, \cdots$ 成立. 这里

$$
c_{r}(\alpha)=r \sum_{\mu=0}^{r-1}(-1)^{r}\left(\begin{array}{c}
r-1 \\
\mu
\end{array}\right) \frac{1}{\mu+1-\alpha} .
$$

Л. П. Фалалеев 在 1981 年将 $\tilde{\Delta}^{\prime}(\alpha)$ 的结果推 广到 $r \geqslant 3$ (不必须是整) 的情形.

本文继续以上的工作, 将 $\Delta_{n}^{\prime}(\alpha)$ 的结果推广到 $r \geqslant 1$ 的一切实数, 证明和得到了

定理 对于 $r \geqslant 1$ 的一切实数, 当 $n \rightarrow \infty$ 时有

$$
\Delta_{n}^{\prime}(\alpha)=\left\{\begin{aligned}
\frac{2}{\pi n^{\alpha}} \Gamma(\alpha) \sin \frac{\alpha \pi}{2} & \frac{\Gamma(1-\alpha) \Gamma(r+1)}{\Gamma(r-\alpha+1)} \\
+o\left(\frac{1}{n}\right), & \text { 当 } 0<\alpha<1, \\
\frac{2 r}{n \pi} \ln n+o\left(\frac{1}{n}\right), & \text { 当 } \alpha=1 .
\end{aligned}\right.
$$

张谊宾

（河北师范学院，石家庄） 